\title{
E-business: Building security on trust
}

Received: 4th June, 2001

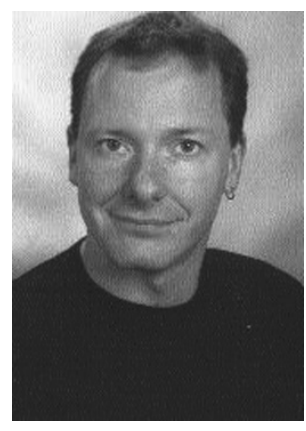

\section{Dr Sachar Paulus}

is Head of the Product Management Team for Security and Directory Services at SAP AG. Before joining SAP he worked as a consultant for SECUDE GmbH and KOBIL Systems $\mathrm{GmbH}$, as well as doing research work at the Institute of Experimental Mathematics at Essen University and at the Department for Theoretical Computer Science at Darmstadt University of Technology. He has a PhD in number theory. Sachar has been active in the area of cryptography since 1990 and has written a number of publications in international magazines and conferences on cryptography and data security. His main areas of interest are PKI and Smart Card technology as well as business cases for security applications and IT risk management. He is speaking at the ISSE 2001 conference in London in September on the subject of this paper.

Abstract Any enterprise moving its business to the Web is inevitably faced with an increase in data security risks. Security processes must first be documented and then implemented in the form of a comprehensive security architecture, paying particular attention to four key areas: efficient user and role management, secure system management, and realisation of the trust relationships and of security mechanisms at the application level.

Any enterprise moving its business to the Web is inevitably faced with an increase in data security risks. Doing e-business usually entails the migration to the Internet of entire processes and their associated data - confidential data that must not under any circumstances be allowed to fall into the hands of competitors. All possible security risks must therefore be identified as early on as possible and appropriate measures taken. In many countries this is already a legal requirement. Germany, for example, passed a law in 1998 - the KonTraG (Gesetz zur Kontrolle und Transparenz im Unternehmensbereich) — that requires companies to implement a monitoring system to give early warnings of any threatening developments.

Across-the-board risk management may not be popular, but it has become indispensable.

Information is a valuable commodity
- not only for one's own enterprise but also for competitors. Four crucial factors determine the security of sensitive data: integrity (data cannot be manipulated), authenticity (the originator of the data can be identified with 100 per cent certainty); confidentiality (data are accessible only by the 'right' persons); and availability (data are always accessible when required). E-business security demands that each of these properties be guaranteed.

The cost of security measures must be commercially justifiable, however. Risk analysis is essential in order to estimate both the value of the information and the likelihood of a security leak. This analysis provides a guideline for the size of investment that can be justified for protective mechanisms. After risk analysis the next step is to investigate each of the mechanisms under consideration, and in particular to assess their practicability. 


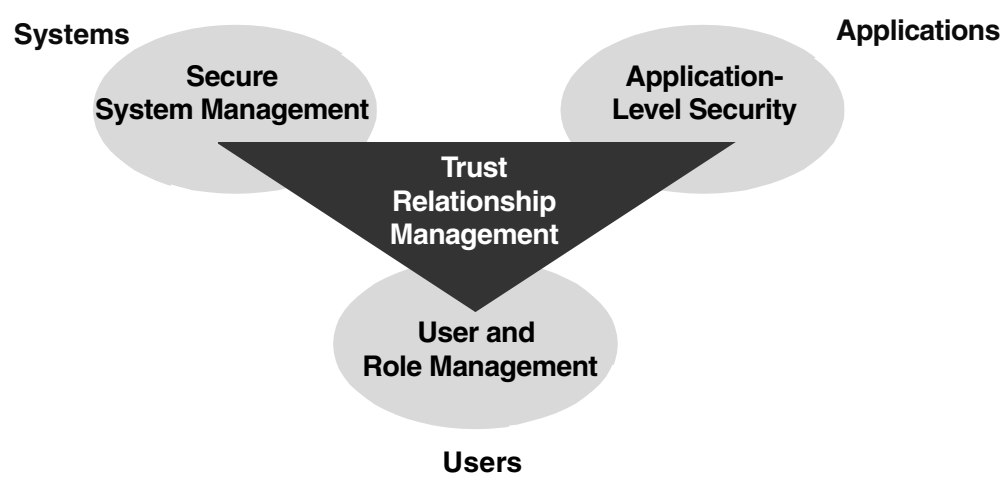

Figure 1 Trust relationships in e-business

Since any given mechanism is likely to have unavoidable side-effects on certain business processes, each specific proposal should be communicated within the corporate hierarchy, in order to ensure that senior management is aware of and agrees to the consequences of any decision taken. The investigations need to be repeated at regular intervals in order to ensure continuous data protection. The final result of this process ought to be a fairly detailed security policy.

The security policy needs to take account of every possible aspect of security within an enterprise, because an absolutely watertight concept is an essential prerequisite for the trust-based relationships that are central to e-business. Processes must first be documented and then implemented in the form of a comprehensive security architecture, paying particular attention to four key areas: efficient user and role management, secure system management, and realisation of the trust relationships and security mechanisms at the application level.

\section{USER AND ROLE MANAGEMENT}

The business processes typical of e-business are creating exciting new opportunities for enterprises: customers and business partners can be integrated seamlessly into business processes, while employees can be granted significant levels of responsibility within these processes. But how can a business make sure that users, whether internal or external, cannot gain access to information that they should not see? And how can employees' roles and authorisations be managed in a way that is secure, efficient and application independent?

The Internet economy characterised as it is by the complex system environments required by electronic business processes and by corporate networks that are necessarily open to outside access — is changing the way that user and authorisation management is done. More than ever, a high priority is being attached to such things as simple, centralised administrative processes, the integration of external users into authorisation processes, a universal role concept that can be applied to every application used in the enterprise and the integration of directory services via enterprise portals.

In many system environments, user information is dispersed across several different systems: e-mail, telephone and application systems are typical examples. 


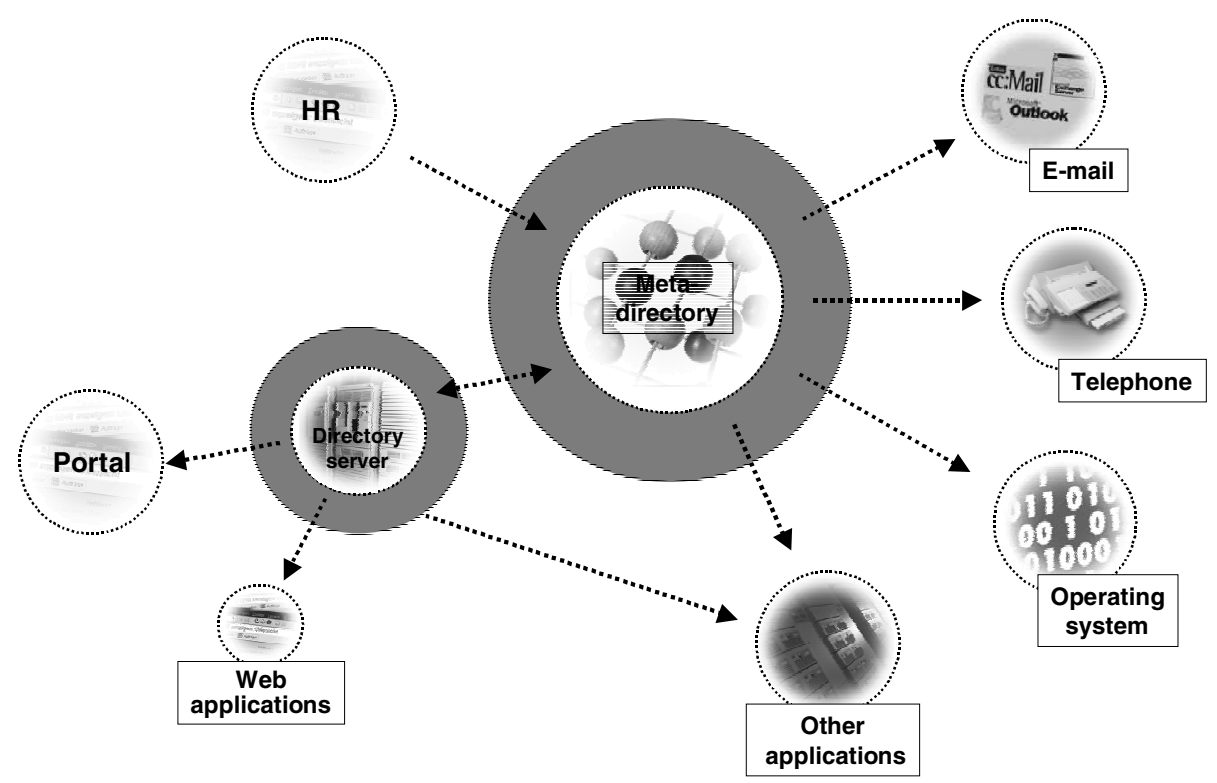

Figure 2 User management based on a directory service

Thus, a single enterprise will often have a number of directories containing the same or at least similar data. One way of avoiding this kind of data redundancy is effectively to combine each of these user data directories into a central meta-directory for the whole enterprise. This central directory obtains user data directly from the human resource system and passes it on to all other connected systems as required - eg to messaging, telephone and application systems.

Information about roles and responsibilities can also be stored in this way. Implementing a directory service creates a 'single point of administration' for user management. Minimising data redundancy and centralising administrative processes allows the highest levels of both efficiency and security to be achieved.

The role concept needs to make a clear distinction between roles and authorisations. This will help keep the concept independent of specific applications and will simplify administration. A role consists of a number of logical services - for example, the creation of customer orders or order billing — which together define an employee's activity profile. These logical services are then allocated to physical services such as those for enabling access to transactions or to reports in application systems. Thus roles are defined according to relevant activities, in other words 'bottom-up', and on a department-by-department basis. Combining employee authorisations into the same roles would result in a very complex administrative process, since authorisations are usually defined along the lines of the organisational structure and according to the user's position in the organisation - in other words 'top-down'. It is, moreover, common for authorisations to be assigned either centrally, for example in the human resource organisation management, or in individual applications, in controlling or financial accounting, for example. 


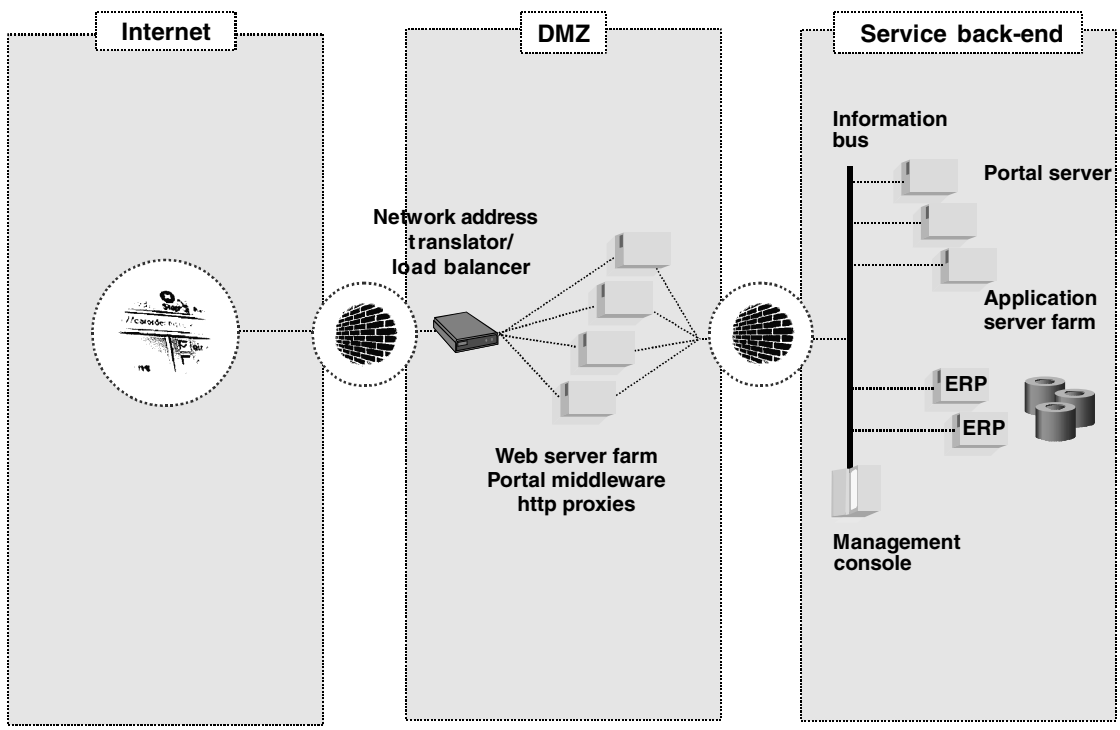

Figure 3 A secure network architecture

\section{SECURE SYSTEM MANAGEMENT}

The creation of secure network configurations is a well-understood science - based on the principles of defining network segments and configuring firewalls. The trend toward opening corporate networks to outside access, however, has made the elimination of all security gaps increasingly tricky. A network should consist of a set of security zones together with a small number of connections between these zones, each protected by a firewall. External access to the corporate network is provided by means of so-called demilitarised zones (DMZ), which use a combination of external and internal firewalls. This ensures that access to the information systems is only possible from certain zones and using specified services and protocols.

Together with appropriate mechanisms for authenticating system components and for encrypting communications between servers, this provides a good level of protection. For this reason the use of HTTP on the standard Internet protocol known as Secure Sockets Layer (HTTPS $=$ HTTP on SSL) is strongly recommended for all Internet communication. HTTPS is now supported together with strong encryption by all commonly used web servers and browsers worldwide. It is also possible to integrate intrusion detection systems into system management. These record all unusual system activity and detect attempts at unauthorised access.

\section{BUILDING TRUST ON RELIABLE AUTHENTICATION}

Authentication mechanisms are becoming increasingly hard to manage in complex e-business system environments. In environments with a large number of systems, users can no longer be expected to remember the multiplicity of passwords needed. Procedures such as single sign-on and authentication delegation have become practically indispensable for an e-business environment. 


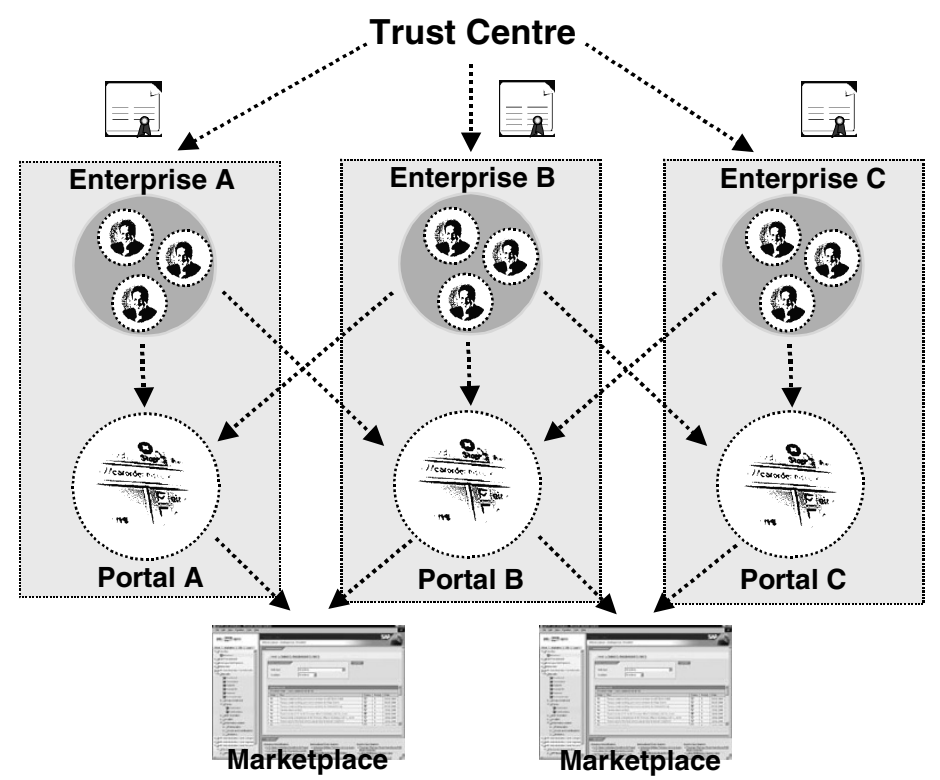

Figure 4 Secure access to external and internal web sites

Because systems are increasingly designed for internal use by a company's own staff as well as external use by customers and partners, the mechanism used for identifying and logging on users must be both secure and easy to use. Digital certificates, issued by a recognised 'trust centre' and embodying standardised authentication processes, offer a very high level of security for electronic transactions. A trustworthy registration authority is set up, with which all users must register. Ideally this is integrated directly into the user management system and can pass user data straight to the trust centre. For each user the trust centre creates a digital identity consisting of two keys, one public and the other private. The digital certificate contains the name of the user and the public key of the digital identity, while the private key is known only to the owner of the certificate. The certificate can now be used to perform the authentication required at portals or at virtual marketplaces.

\section{SECURITY MECHANISMS AT THE APPLICATION LEVEL}

Collaborative business processes are frequently distributed across a number of interlinked systems. For this reason, detailed authorisation management alone cannot guarantee a secure, audit-proof environment. When processes are executed in distributed system environments, the trust relationship information must be attached directly to the data so that records can be kept to prove that the data were used correctly. Companies can achieve this by using digital signatures. Collaborative transactions are allocated a number of electronic properties, and these help guarantee both data integrity and authenticity over the entire lifespan of the transaction.

Since the technology required for this cannot be made available centrally, it is integrated into the application processes. A digital signature equips data - for example, the details of an online order - with additional information that helps 


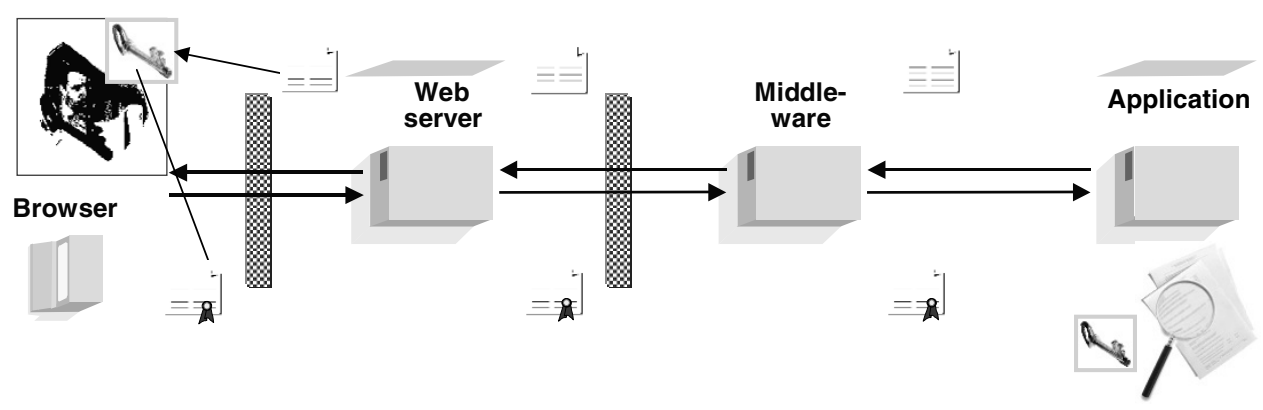

Figure 5 Transaction security based on digital signatures

guarantee the integrity of the transaction. Each system through which the data pass and which has the functions required for verifying digital signatures can check this information. Verification can be performed at a number of different levels. Factors such as the type of certificate or the issuing trust centre, for example, can be used to determine whether or not the signature is accepted; the validity of the certificate at the time the signature was made can also be checked. Business processes involving digitally-signed transactions require a fall-back configuration for instances where a transaction is aborted following a negative verification.

The application itself is responsible for security in other areas too, for example in the statutory forms of digital signatures for bid invitations and contracts, in electronic payment procedures, e-mail encryption or steganography.

\section{THE SECURITY CHALLENGE}

The growth of Internet usage has made the protection of corporate systems and data - no trivial task even back in the days of closed-system environments into a highly significant challenge.

Whereas system-oriented security mechanisms were generally adequate in the past, the mechanisms must now be implemented at the level of individual transactions. If, however, an enterprise ensures that its security policy takes adequate account of the four areas of security architecture described here, the exciting new opportunities offered by e-business can be exploited while avoiding unnecessary security risks. 